\title{
Alternative Genetic Models for the Inheritance of the Phenylthiocarbamide Taste Deficiency
}

\author{
Jane M. Olson, Michael Boehnke, Katherine Neiswanger, Alex F. Roche, and \\ Roger M. Siervogel
}

Department of Biostatistics, University of Michigan, Ann Arbor (J.M.O., M.B.); Departments of Psychiatry and Human Genetics, Western Psychiatric Institute and Clinic, University of Pittsburgh (K.N.); Division of Human Biology, Department of Pediatrics, Wright State University School of Medicine, Yellow Springs, Ohio (A.F.R., R.M.S.)

\begin{abstract}
Pedigree segregation analysis was used to examine several one- and two-locus models of the inheritance of phenylthiocarbamide (PTC) taste deficiency that extend the traditional one-locus recessive model by the addition of either another allele or another locus, and in some cases predict two types of nontasters. These models allow nontaster by nontaster matings to produce taster offspring, consistent with our data and several previous studies which use the Harris and Kalmus [Annals of Eugenics 15:24-32, 1949] dilution method. The models fit our data set of 1,152 individuals from 120 families significantly better than the one-locus recessive model. The best fit was obtained with a two-locus model in which one locus controls PTC tasting and the other locus controls a more general taste ability. This model is consistent with research on the physiology of PTC tasting and with results from genetic linkage studies. Further study is suggested to evaluate better the accuracy of the proposed model.
\end{abstract}

Key words: PTC, two-locus models, complex segregation analysis

\section{INTRODUCTION}

Since its discovery in 1931 by A.L. Fox, primary interest in the phenylthiocarbamide (PTC) taste deficiency has centered on its role as a classic example of autosomal recessive inheritance. Recent research in taste physiology suggests that PTC taste

Received for publication November 29, 1988; revision accepted February 14, 1989.

Address reprint requests to Michael Boehnke, Ph.D., Department of Biostatistics, School of Public Health, University of Michigan, 109 South Observatory, Ann Arbor, MI 48109.

(C) 1989 Alan R. Liss, Inc. 
deficiency may also serve as a useful marker for more general taste sensitivity [Bartoshuk, 1979; Gent and Bartoshuk, 1983].

Several early genetic investigations [Snyder, 1931, 1932; Blakeslee and Salmon, 1931; Blakeslee, 1932; Lee, 1934] using early PTC testing methods, primarily crystals, concluded that the inability to taste PTC is inherited through a single genetic locus as an autosomal recessive. Later work using the Harris and Kalmus [1949] dilution method for PTC testing has questioned this conclusion. Although two studies [Merton, 1958; Rao and Morton, 1977] support the one-locus recessive model, Das [1958], using family data, failed to confirm the one-locus recessive model and suggested an incomplete dominance model. Using sib-pair analyses, Harris and Kalmus [1951], Das [1956], and Merton [1958] all found significant deviations from the one-locus recessive model. More recently, Reddy and Rao [1989] found evidence for polygenes and for incomplete dominance at the major locus. These results suggest that, in addition to a major PTC locus, the dilution method may be measuring tasting variability to which the original crystal method was insensitive.

Other lines of investigation have called into question the adequacy of a one-locus, two-allele model. First, there are consistent reports of taster offspring resulting from nontaster by nontaster matings [Das, 1958; Merton, 1958]. Second, physiologic evidence [Hartmann, 1939; Kalmus, 1958; Frank and Korchmar, 1985] suggests that there may be at least two types of PTC taste deficiency: a specific PTC deficiency and a more general taste deficiency. Third, several studies of linkage with the Kell blood group [Conneally et al., 1976; see summary in Spence et al., 1984] give conflicting results and may suggest locus heterogeneity.

The present analysis was undertaken to examine several one- and two-locus models of PTC inheritance. We postulate that the traditional one-locus recessive model is substantially accurate but requires the addition of either a third allele or a second locus to explain the inconsistencies observed using the dilution method. Further, we consider three models that distinguish two types of nontasters in accordance with the physiologic evidence. The methods employed allow for the treatment of PTC tasting ability as a quantitative trait while obtaining a maximum likelihood fit to a particular genetic model. We find that several two-locus models and two one-locus, three-allele models provide a substantially better fit to the PTC data than the traditional one-locus recessive model. The best fit is obtained by a two-locus model that predicts two types of nontasters.

\section{MATERIALS AND METHODS}

The sample consisted of families living in southern Ohio and enrolled in the Fels Longitudinal Growth Study [Spence et al., 1984; Roche et al., 1988]. The 120 families reported here were randomly sampled with respect to PTC tasting status and include 1,152 individuals tested for PTC tasting ability between 1970 and 1974. PTC tasting was assessed using the method of Harris and Kalmus [1949], with the slight modification that the 14th (most dilute) solution was eliminated. Blood marker data were available for approximately one-half of the individuals, making it possible to test for nonpaternity in cases where taster offspring resulted from nontaster by nontaster matings. The markers included ABO, RH, P1, KEL, SE, MNS, FY, JK, XG, HP, BF, GC, ACP, C3, ADA, ORM, AG, and PGM1. 
Paternity testing was carried out using the computer program MENDEL [Lange and Boehnke, 1983; Lange et al., 1988], which computes both the paternity index and the nonexclusion probability. The paternity index [Essen-Moeller, 1938] is the ratio of the probability of a parent-offspring trio assuming that the putative father is the real father to the probability of the trio assuming that the putative father is a randomly chosen male. The nonexclusion probability [see, for example, Lange et al., 1986] is the probability that an unrelated male would not be excluded by at least one of the markers tested conditional on the phenotypes of the mother and offspring; it is a more conservative and less controversial measure of paternity.

Pedigree segregation analysis was also carried out using MENDEL with penetrance routines specifically tailored to each genetic model. MENDEL calculates pedigree likelihoods for genetic models involving a small number of genetic loci; the traits modeled may be quantitative or qualitative. MENDEL uses greedy-type algorithms for pedigree likelihood calculations and a variable metric method for iterative maximum likelihood estimation [Lange and Boehnke, 1983; Lange et al., 1988].

The relative adequacy of the various models was evaluated by comparing their $\log$ likelihoods. Statistical tests of hypothesis using the likelihood ratio statistic are possible when one model is nested within another. In cases where none of the null hypothesis values of the parameters are on the boundary of the parameter space, the asymptotic distribution of the likelihood ratio statistic is chi-square with degrees of freedom equal to the difference in the number of independent parameters between the two models. When the null hypothesis value of the parameter of interest is on the boundary of the parameter space, the asymptotic distribution of the likelihood ratio statistic is a 50:50 mixture of a point mass at 0 and a chi-square distribution on 1 degree of freedom [Self and Liang, 1987]. The significance level of the mixture distribution is one-half that which would result if the same statistic were compared to a chi-square distribution on 1 degree of freedom. Asymptotic theory standard errors were calculated by inverting minus the Hessian and taking square roots of the diagonal elements of this inverse matrix.

The 14 discrete PTC thresholds are assumed to span the range of values of a continuous trait. Distributions of threshold scores are generally bimodal corresponding to the qualitative phenotypes of nontaster and taster. The population distribution of quantitative PTC scores was therefore modeled as a mixture of normals. Initial estimates for the means and variances of the component distributions and their proportions were obtained by fitting mixtures of normal distributions to the PTC scores assuming they represent independent observations [Day, 1969]. For two-locus models, loci were assumed to be unlinked.

Several one- and two-locus models were fit to the PTC family data. Models were chosen based on 1) the assumption that the traditional one-locus model is substantially accurate but requires extension to explain the observed data; and 2) the suggestion that there exists more than one type of nontaster. Parameters estimated for all models were allele frequencies $(p)$ at each locus and means $(\mu)$ and standard deviations $(\sigma)$ for each genotype-specific normal distribution.

The one-locus models tested were the traditional two-allele recessive model (1LR), a three-allele model (ILR3A) predicting two distributions (taster and nontaster), and a three-allele model (1LR3A-3D) predicting three distributions (one taster and two nontaster) (Table I). In general, the taster allele ( $T$ ) is assumed to be dominant to the nontaster 
TABLE I. Description of PTC Genetic Models*

\begin{tabular}{|c|c|c|c|c|c|c|}
\hline \multirow[b]{2}{*}{ Model } & \multicolumn{2}{|c|}{$\begin{array}{l}\text { No. of } \\
\text { alleles }\end{array}$} & \multirow{2}{*}{$\begin{array}{c}\text { No. of } \\
\text { distributions }\end{array}$} & \multicolumn{3}{|c|}{$\begin{array}{c}\text { Genotype/phenotype } \\
\text { correspondence }\end{array}$} \\
\hline & Locus 1 & Locus 2 & & NT(1) & NT(2) & $\mathrm{T}$ \\
\hline \multicolumn{7}{|c|}{ One-locus models ${ }^{\mathrm{a}}$} \\
\hline $1 \mathrm{LR}$ & 2 & - & 2 & $\mathrm{tt}$ & & $\mathrm{T}-$ \\
\hline 1LR3A & 3 & - & 2 & $S-, \mathrm{tt}$ & & $\mathrm{TT}, \mathrm{Tt}$ \\
\hline 1LR3A-3D & 3 & - & 3 & $S-$ & $\mathrm{tt}$ & $\mathrm{TT}, \mathrm{Tt}$ \\
\hline \multicolumn{7}{|c|}{ Two-locus models } \\
\hline $2 \mathrm{LR}+\mathrm{D}$ & 2 & 2 & 2 & $\mathrm{ttS}-$ & & $\mathrm{T}---,--\mathrm{ss}$ \\
\hline 2LR/D & 2 & 2 & 2 & $\mathfrak{t}--,--S-$ & & $T-s s$ \\
\hline 2LR/D-3D & 2 & 2 & 3 & $--S-$ & ttss & $T-s s$ \\
\hline 2LR/R & 2 & 2 & 2 & $\mathrm{tt}--,--\mathrm{ss}$ & & $T-S-$ \\
\hline 2LR/R-3D & 2 & 2 & 3 & $--s s$ & $\mathrm{ttS}-$ & $T-S-$ \\
\hline
\end{tabular}

*." _ "in the genotype/distribution correspondence implies either allele at the given locus. For example, $\mathrm{T}$ includes genotypes TT and Tt. " + "in a model name signifies "and"; "," in a model name signifies "or." ${ }^{a}$ For the one-locus models, $\mathrm{S}$ is dominant to both $\mathrm{T}$ and $\mathrm{t}$.

allele (t). In the one-locus, three-allele models, a second nontaster allele (S) is assumed to be dominant to both the $\mathrm{T}$ and $\mathrm{t}$ alleles, making taster offspring possible from the mating of two nontaster individuals. A model with another recessive allele would not allow this outcome and was not tested.

Two-locus models considered included all six possible two-allele dominant-recessive relationships producing nontasters. In general, at the first $(\mathrm{T})$ locus, $\mathrm{T}$ is dominant to $\mathrm{t}$; at the second (S) locus, $\mathrm{S}$ is dominant to s. The two-locus models considered included recessive at both loci, dominant at both loci, recessive at either locus (2LR/R), dominant at either locus, recessive at the first (T) locus and dominant at the second (S) locus $(2 \mathrm{LR}+\mathrm{D})$, and recessive at the first $(\mathrm{T})$ locus or dominant at the second (S) locus (2LR/D) (Table I). The recessive-at-both-loci model was excluded since nontaster by nontaster matings would produce only nontaster offspring, inconsistent with the data. The dominant-at-either-locus model was excluded because taster by taster matings would produce only taster offspring, again inconsistent with the data. The dominantat-both-loci model was not considered because it is not the result of the addition of a second locus or a third allele to the one-locus recessive model.

The three remaining two-locus models were fit assuming two distributions (taster and nontaster) and were then extended to three-distribution models if the model suggested more than one type of nontaster (Table I). The three-distribution recessive-ordominant model (2LR/D-3D) defines PTC nontasters as having genotype ttss and general nontasters as having genotypes of the form - - $-\mathrm{S}-$, where " - " implies that either allele may be present. The three-distribution either-recessive model (2LR/R-3D) defines PTC nontasters as $\mathrm{ttS}$ - and general nontasters as - - ss. In both cases, the presence or absence of the general nontaster phenotype is controlled by the genotype at the second (S) locus alone; PTC nontasters result only when the genotype at the first (T) locus is tt but that at the second (S) locus does not result in a general nontaster. The recessive-and-dominant model was not extended because only one type of nontaster $(\mathrm{ttS}-)$ is predicted. 


\section{RESULTS}

A histogram of PTC scores for the entire sample of 1,152 individuals is shown in Figure 1. In a preliminary analysis, a subset of the data consisting of 402 parent-offspring trios from 175 nuclear families was examined for inheritance patterns. Trios were included if PTC scores were available on all three individuals. As more than one trio was often chosen from the same nuclear or extended family, the trios are not all independent. The observed antimode of 5 was used to classify individuals as either nontaster (NT: PTC $\leqslant 5$ ) or taster (T: PTC $>5$ ); no such dichotomization of the quantitative PTC scores was required for subsequent pedigree analysis using MENDEL.

The observed and expected numbers of offspring from the three possible mating types $(\mathrm{T} \times \mathrm{T}, \mathrm{T} \times \mathrm{NT}$, and $\mathrm{NT} \times \mathrm{NT})$ are shown in Table II. Expected numbers of taster and nontaster offspring were calculated using Snyder's [1932] ratios. There is a significant excess of taster offspring as compared to the number expected under the one-locus recessive model. Of particular note are the 18 tasters among the 44 offspring of NT $\times$ NT matings. This result is inconsistent with the autosomal recessive model, which predicts no taster offspring from this mating type. Changing the cutpoint for $\mathrm{T}$ and NT status (i.e., using $\leqslant 4$ or $\leqslant 6$ ) did not substantially affect the proportion of such offspring.

To take into account the possible effect of age on PTC sensitivity [Harris and Kalmus, 1949; Kalmus and Trotter, 1962], we performed a linear regression of PTC

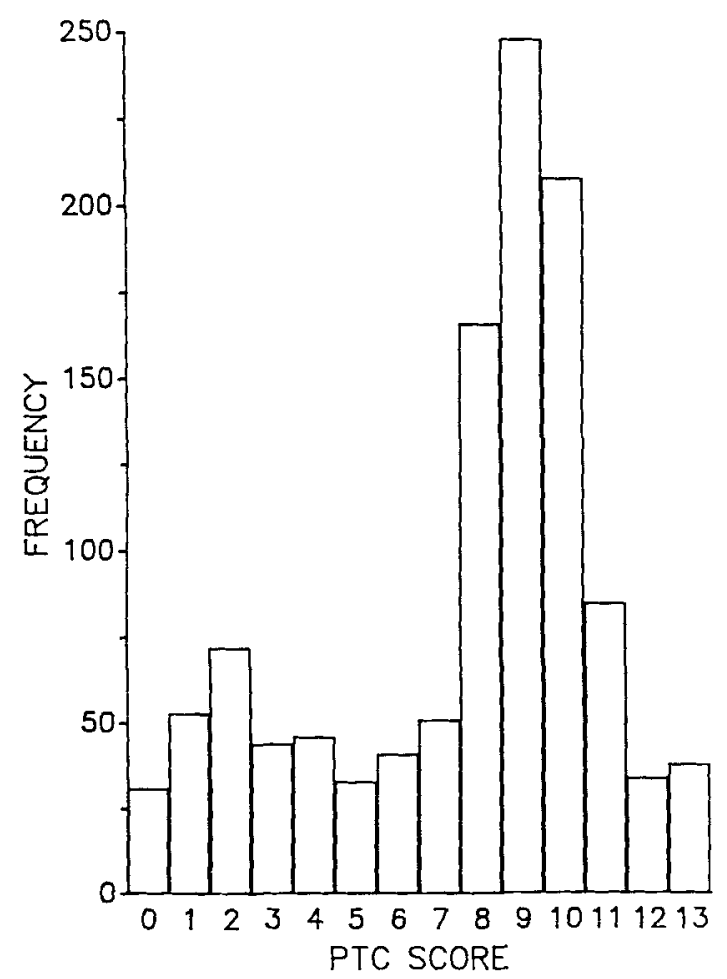

Fig. 1. Distribution of PTC scores for 1,152 subjects. 
TABLE II. Inheritance Patterns of 402 Parent/Offspring Trios From 175 Nuclear Families*

\begin{tabular}{lcccc} 
& \multicolumn{3}{c}{ Mating type } & \\
\cline { 2 - 4 } Offspring type & $\mathrm{T} \times \mathrm{T}$ & $\mathrm{T} \times \mathrm{NT}$ & $\mathrm{NT} \times \mathrm{NT}$ & Total \\
\hline NT & 19 & 28 & 26 & 73 \\
& $(23.93)^{\mathrm{a}}$ & $(48.60)$ & $(44)$ & $(116.53)$ \\
$\mathrm{T}$ & 194 & 117 & 18 & 329 \\
& $(189.07)$ & $(96.40)$ & $(0)$ & $(285.47)$ \\
Total & 213 & 145 & 44 & 402 \\
\hline
\end{tabular}

${ }^{*}$ Chi-square for $\mathrm{T} \times \mathrm{T}$ and $\mathrm{T} \times \mathrm{NT}$ matings $=14.28, \mathrm{df}=2, P<.001$.

${ }^{a}(\quad)=$ Expected frequencies assuming the one-locus recessive (ILR) model using nontaster allele frequency estimate of $\sqrt{91 / 358}=0.5042$ from the No. of nontasters among the unrelated individuals in the sample.

score on age using the entire data set $\left(\mathrm{R}^{2}=0.03\right.$, slope $\left.=-0.031, P<.0001\right)$ and reexamined the trio data using residual scores from the regression (Table III). Ages were unavailable for one member of three of the trios. As young children are often considered unreliable in PTC testing, this analysis was also repeated excluding children under 10 years of age.

The antimode $x$ for the distribution of residuals was estimated by finding the value $x$ that minimizes misclassification given a mixture of two normal distributions. This misclassification was estimated as

$$
\mathrm{p}\left\{1-\Phi\left[\left(\mathrm{x}-\mu_{1}\right) / \sigma_{1}\right]\right\}+(1-\mathrm{p}) \Phi\left[\left(\mathrm{x}-\mu_{2}\right) / \sigma_{2}\right]
$$

using estimates for $\mathrm{p}, \mu_{1}, \mu_{2}, \sigma_{1}$, and $\sigma_{2}$ generated by fitting a mixture of two normal distributions to the residuals, where $\Phi$ is the standard normal cumulative distribution function. Although linear regression on age reduced the number of taster offspring resulting from NT $\times$ NT matings from 18 to 14, these results remain inconsistent with the autosomal recessive model (Table III). Further, expected numbers of taster offspring remain significantly larger than expected for the $T \times T$ and $T \times N T$ matings. The results of the analysis excluding young children were analogous and are not shown.

The families of the 14 taster offspring resulting from NT $\times$ NT matings were examined for possible nonpaternity. Blood marker data were available on 11 of the 14; the results are presented in Table IV, along with the age of each family member. Blood

TABLE III. Inheritance Patterns of 399 Parent/Offspring Trios From 175 Nuclear Families (Residuals)*

\begin{tabular}{lcccc} 
& \multicolumn{3}{c}{ Mating type } & Total \\
\cline { 2 - 4 } Offspring type & $\mathrm{T} \times \mathrm{T}$ & $\mathrm{T} \times \mathrm{NT}$ & $\mathrm{NT} \times \mathrm{NT}$ & 74 \\
$\mathrm{NT}$ & 21 & 29 & 24 & $(109.65)$ \\
& $(25.69)^{\mathrm{a}}$ & $(45.96)$ & $(38)$ & 325 \\
$\mathrm{~T}$ & 204 & 107 & 14 & $(289.35)$ \\
& $(199.31)$ & $(90.04)$ & $(0)$ & 399 \\
\hline
\end{tabular}

${ }^{*}$ Chi-square for $\mathrm{T} \times \mathrm{T}$ and $\mathrm{T} \times \mathrm{NT}$ matings $=10.42, \mathrm{df}=2, P<.005$.

${ }^{a}()=$ Expected frequencies assuming the one-locus recessive (ILR) model using nontaster allele frequency estimate of $\sqrt{93 / 357}=0.5104$ from the No. of nontasters among the unrelated individuals in the sample. 
TABLE IV. Paternity Testing of Taster Offspring Produced by Nontaster by Nontaster Matings

\begin{tabular}{|c|c|c|c|c|c|c|c|c|}
\hline PTC & $\begin{array}{l}\text { Father } \\
\text { (age in years) }\end{array}$ & PTC & $\begin{array}{l}\text { Mother } \\
\text { (age in years) }\end{array}$ & PTC & $\begin{array}{l}\text { Offspring } \\
\text { (age in years) }\end{array}$ & $\begin{array}{c}\text { Paternity } \\
\text { index }\end{array}$ & $\begin{array}{c}\text { Nonexclusion } \\
\text { probability }\end{array}$ & $\begin{array}{c}\text { No. of blood } \\
\text { markers }\end{array}$ \\
\hline 1 & (40) & 3 & (39) & 9 & (17) & 49 & .031 & 10 \\
\hline 1 & (62) & 1 & (61) & 8 & (32) & 11 & .190 & 12 \\
\hline 0 & (71) & 1 & $(67)$ & 8 & (26) & 54 & .038 & 15 \\
\hline 1 & (65) & 4 & (72) & 9 & (41) & 126 & .070 & 14 \\
\hline 1 & (37) & 2 & (38) & 8 & (14) & 54 & .107 & 12 \\
\hline 1 & (37) & 2 & (38) & 11 & (4) & 2 & .668 & 6 \\
\hline 2 & (45) & 2 & (44) & 6 & (18) & 9 & .144 & 9 \\
\hline 0 & (65) & 4 & (53) & 11 & (22) & 3 & .365 & 10 \\
\hline 1 & $(62)$ & 1 & (61) & 7 & (33) & \multicolumn{2}{|c|}{ Incompatibility at PGMI } & 14 \\
\hline 1 & (72) & 4 & (65) & 8 & (36) & \multicolumn{2}{|c|}{ Incompatibility at ACP } & 15 \\
\hline 2 & (45) & 2 & (44) & 8 & (15) & \multicolumn{2}{|c|}{ Incompatibility at $\mathrm{ABO}$} & 15 \\
\hline
\end{tabular}

marker incompatibilities were discovered in three of the 14 offspring. In the subsequent pedigree analysis, the three offspring with no blood marker data and the three marker-incompatible offspring were treated as isolated individuals and not as members of their reported pedigrees.

Residuals from the regression of PTC tasting score on age were transformed back to the original scale using the estimated regression equation. The resulting variable was used in the pedigree segregation analysis. Table V shows parameter estimates for the fitted models for the transformed PTC tasting scores. Results using raw PTC scores were similar and are not shown. Results for the two-distribution models with a single common standard deviation were similar to those allowing for 2 standard deviations. For the three-distribution models, models with a single common standard deviation provided much poorer fits to the data, suggesting possible genotype-by-environment interaction or left truncation of the distribution. The one-locus recessive model (1LR) and the two-locus, three-distribution either-recessive model (2LR/R-3D) were reana-

TABLE V. Parameter Estimates and Log Likelihoods for the PTC Genetic Models

\begin{tabular}{|c|c|c|c|c|c|c|c|c|c|}
\hline \multirow{2}{*}{$\begin{array}{l}\text { Genetic } \\
\text { Model }\end{array}$} & \multicolumn{8}{|c|}{ Parameter estimates } & \multirow[b]{2}{*}{$2 \log L+5376.38$} \\
\hline & $\rho_{1}$ & $\rho_{2}$ & $\mu_{1}$ & $\mu_{2}$ & $\mu_{3}$ & $\sigma_{1}$ & $\underline{\sigma}_{2}$ & $\sigma_{3}$ & \\
\hline \multicolumn{10}{|l|}{ None } \\
\hline \multicolumn{2}{|l|}{2 distributions } & & 2.47 & & 9.20 & 1.44 & & 1.62 & \\
\hline \multicolumn{2}{|c|}{3 distributions } & & 1.55 & 3.87 & 9.25 & 0.80 & 1.42 & 1.53 & \\
\hline \multicolumn{10}{|c|}{ One-locus models } \\
\hline $1 \mathrm{LR}$ & 0.484 & & 2.78 & & 9.27 & 1.79 & & 1.58 & 0.00 \\
\hline 1LR3A & 0.553 & 0.405 & 2.47 & & 9.20 & 1.45 & & 1.63 & 35.16 \\
\hline 1LR3A-3D & 0.528 & 0.419 & 1.84 & 3.51 & 9.32 & 1.03 & 1.93 & 1.53 & 47.65 \\
\hline \multicolumn{10}{|c|}{ Two-locus models } \\
\hline $2 \mathrm{LR}+\mathrm{D}$ & 0.350 & 0.349 & 2.48 & & 9.21 & 1.46 & & 1.62 & 41.06 \\
\hline 2LR/D & 0.573 & 0.040 & 2.47 & & 9.20 & 1.45 & & 1.62 & 36.23 \\
\hline 2LR/D-3D & 0.543 & 0.041 & 1.59 & 3.22 & 9.29 & 0.87 & 1.74 & 1.55 & 52.00 \\
\hline 2LR/R & 0.693 & 0.594 & 2.47 & & 9.20 & 1.45 & & 1.62 & 31.78 \\
\hline $2 \mathrm{LR} / \mathrm{R}-3 \mathrm{D}^{\mathrm{a}}$ & 0.682 & 0.570 & 1.44 & 3.59 & 9.30 & 0.77 & 1.63 & 1.54 & 73.70 \\
\hline
\end{tabular}

${ }^{\mathrm{a}} \mathrm{Standard}$ errors for this model are $0.027,0.029,0.120,0.247,0.129,0.084,0.170,0.050$, respectively. 
lyzed excluding children under 10 years of age; results were very similar to those in Table V.

Figure 2 shows the nesting structure of the fitted models and corresponding likelihood ratio statistics and significance levels. Models that added a third allele or a second locus of the traditional one-locus recessive model (1LR) provided significantly better fits to the data than did the one-locus recessive model. Among the two-distribution models, the two-locus recessive-and-dominant $(2 \mathrm{LR}+\mathrm{D})$ model had the largest log likelihood by a small margin.

Further, models predicting three distributions fit the data significantly better than corresponding models predicting two distributions (Fig. 2). Among the three-distribution models, the recessive-at-either-locus ( $2 \mathrm{LR} / \mathrm{R}-3 \mathrm{D}$ ) model was best supported by the data. Although the three-distribution models are nonnested and so cannot be compared using a likelihood ratio test, the probability of the data under the 2LR/R-3D model was more than 50,000 times greater than under any other model considered (Table V).

We used the estimates on the allele frequencies for each genetic model considered to compute the predicted overall proportion of nontasters and the predicted proportions of nontaster offspring resulting from $\mathrm{T} \times \mathrm{T}, \mathrm{T} \times \mathrm{NT}$, and $\mathrm{NT} \times \mathrm{NT}$ matings and compared these proportions to those observed in the analysis of the parent-offspring trio data. With the exception of the one-locus recessive model, which does not allow NT $\times$ NT matings to produce tasters, no one model generated predictions that were clearly better or worse than the predictions of the other models.

To assess the relative impact of taster offspring resulting from NT $\times$ NT matings

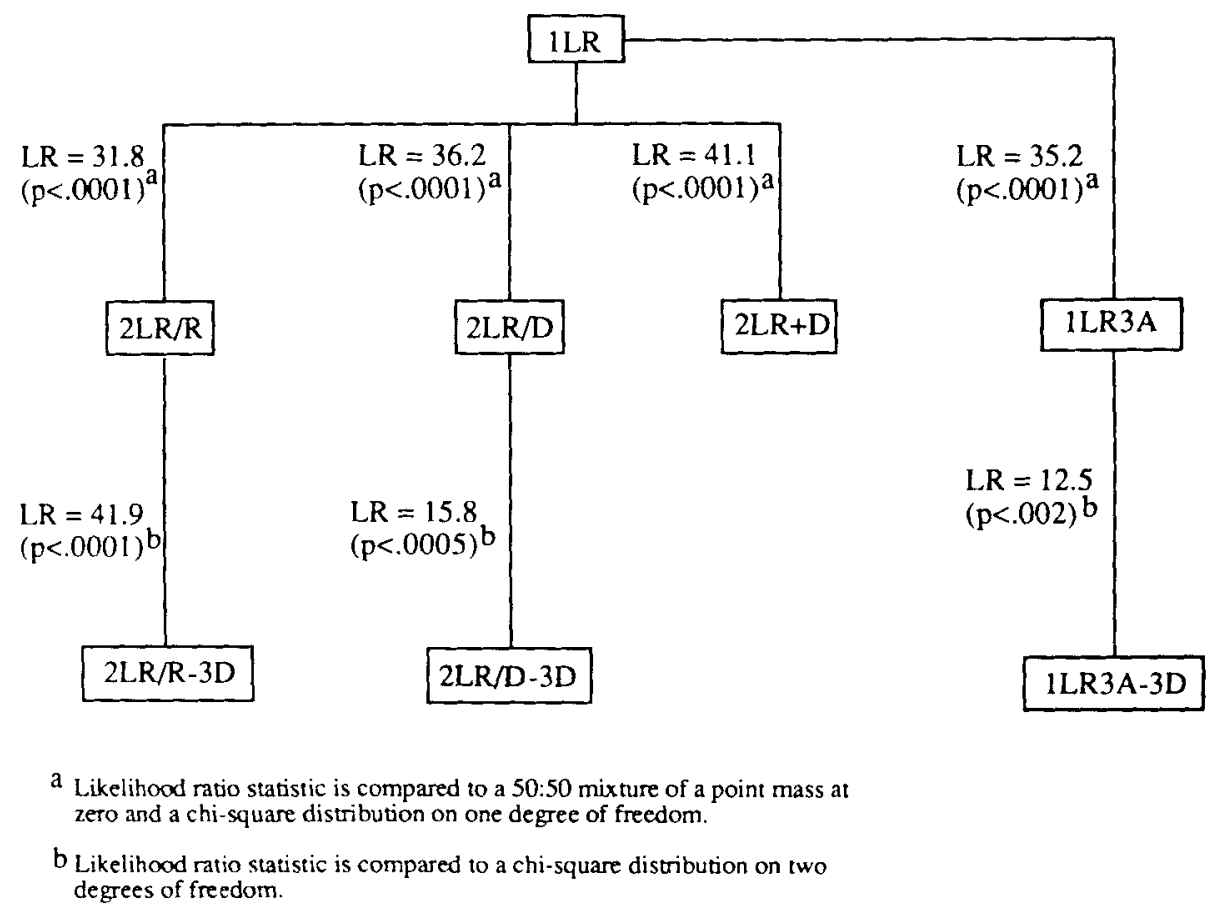

Fig. 2. Hierarchical structure and significance testing of PTC models. 
on these results, log likelihood differences between models for individual pedigrees were examined. Families with such offspring contributed virtually all of the difference in log likelihood between the one-locus recessive model and the other models, suggesting that the two-locus and three-allele models improve on the traditional model largely because they explain the observation that NT $\times$ NT matings produce taster offspring.

\section{DISCUSSION}

The two-locus and one-locus three-allele models presented here provided a better explanation for the inheritance of quantitative PTC tasting score than the traditional one-locus recessive model for the Fels study data we analyzed. The model in which nontasters were recessive at either of two loci and which allowed for two classes of nontasters (2LR/R-3D) was best supported by the data.

Several lines of evidence are consistent with the suggestion of an additional locus or allele. First, most of the support for the one-locus recessive model comes from early studies [Snyder, 1931, 1932; Blakeslee and Salmon, 1931; Blakeslee, 1932; Lee, 1934] that used crystal or limited dilution methods for PTC testing. Several later investigators [Harris and Kalmus, 1951; Das, 1956, 1958; Merton, 1958; Reddy and Rao, 1989] used the Harris and Kalmus [1949] 14-dilution method and observed significant lack of fit to the one-locus recessive model. In addition, Merton noted five taster offspring among 40 offspring of NT $\times$ NT matings; Das [1958] found four taster offspring among 41 offspring of such matings.

Second, linkage heterogeneity has been suggested for PTC based on findings of linkage with the Kell blood group in one study [Conneally et al., 1976] but not in others [see summary in Spence et al., 1984].

Third, there is physiologic evidence suggesting that there may be at least two types of PTC taste deficiency. In an exploratory study, Frank and Korchmar [1985] tested the reaction times of PTC tasters and nontasters to a variety of gustatory stimuli and concluded that some nontasters may have a general reaction time deficiency for a wide range of compounds, while others show normal reaction times to substances other than PTC. Some investigators [Boyd and Boyd, 1937; Lugg and Whyte, 1955] observed considerable variation in the subjective description of the taste of PTC. Other workers [Bartoshuk, 1979; Gent and Bartoshuk, 1983] have noted that PTC tasters have a different overall taste experience than nontasters; it would be of interest to determine if nontasters are uniform in this respect.

Kalmus [1958] studied in some detail the relationship between PTC tasting ability and ability to taste the bitter substances quinine and brucine and used this ability to discriminate further between PTC tasters and nontasters. His sample included a small number of extreme nontasters (PTC score $=0$ ) that were also seriously deficient in ability to taste quinine; otherwise, there was no overall difference between tasters and nontasters in quinine tasting ability.

Hartmann [1939] compared a dilution method for PTC testing to the ability to taste PTC crystals and found that many individuals who were classified as nontasters using the dilution method could taste the crystals and would therefore be classified as tasters using the crystal method. Differences in salivation were postulated to account for this result.

In considering a genetic model for PTC inheritance, therefore, it may be hypoth- 
esized that there is one locus with an allele that affects PTC tasting ability specifically and either a second locus or an additional allele controlling a more general taste ability. This general taste ability may be involved with bitter substances, salivation, access to taste buds, or some other ability. The two types of nontasters may be indistinguishable on an individual basis unless other physiologic measures are recorded. Our results are consistent with a model in which separate distributions corresponding to the two types of nontasters are distinguishable at the population level.

Figure 3 depicts the population distribution of PTC scores suggested by our proposed model of PTC inheritance: the recessive-at-either-locus, three-distribution model that was best supported by the data. Extreme nontasters (estimated as $8.2 \%$ of the population) are represented by the lowest distribution, while other nontasters $(18.5 \%)$ are represented by the middle distribution. Tasters (73.3\%) make up the upper distribution. The sum of these three distributions is represented by the dotted curve.

Several points of caution are worth noting. The first is the higher percentage of nontasters among the parents than the offspring in the 175 nuclear families, even when PTC scores are adjusted for age. In particular, the precentage of nontasters jumps from about $19 \%$ to about $25 \%$ around age 30 years and remains essentially constant thereaf-

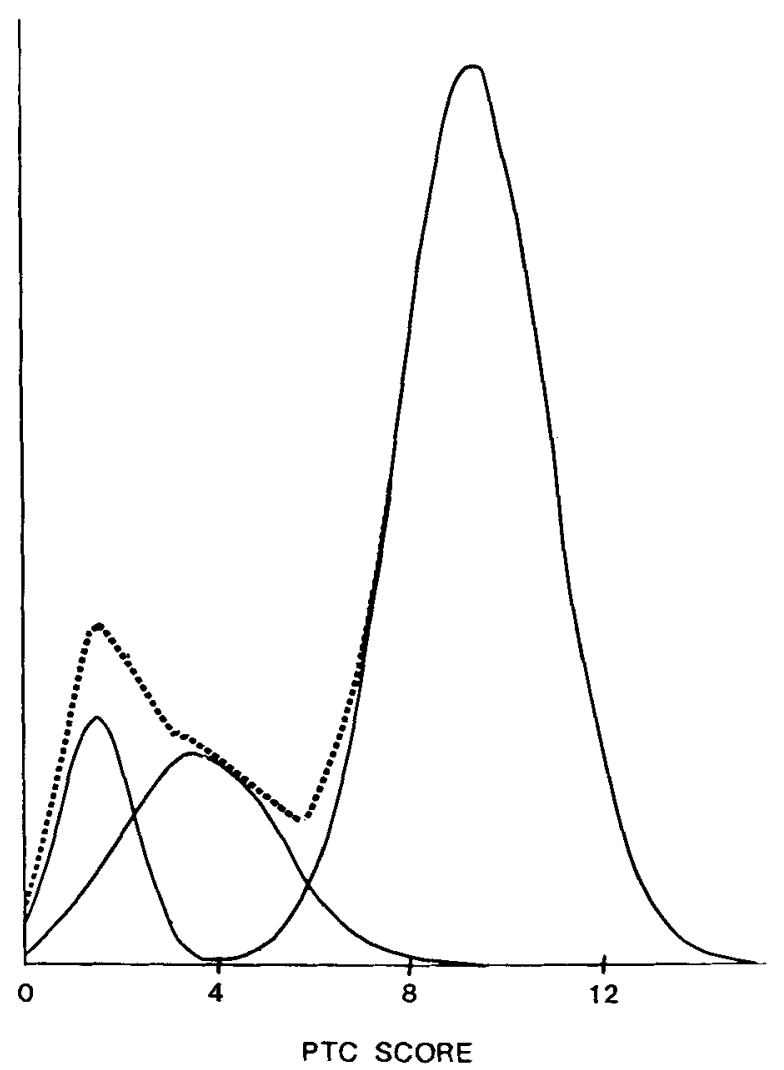

Fig. 3. Population distribution of PTC scores suggested by the 2LR/R-3D model. 
ter. Therefore, the possibility that some adults may have lost their ability to taste PTC needs to be considered; such loss may be either genetic or environmental in origin.

Second, the effects of covariates (for example, smoking) need to be examined more closely to ensure that extraneous sources of variation are accounted for. Third, fitting another family of distributions such as the lognormal or some other skewed family of distributions could result in differences in inference.

Fourth, taster offspring resulting from NT $\times$ NT matings must be examined very carefully. Medical histories of the parents of these offspring were noncontributory in all but one case: one parent had episodes of pneumonia at ages 2 and 25 years. As our data were collected 10-15 years prior to this analysis, it has been possible to repeat PTC testing on only a few of the individuals in a few of the families. Nonetheless, an overall rate of $40 \%$ taster offspring from NT $\times$ NT matings seems too high to be entirely attributed to nonpaternity, inaccurate testing, or parental misclassification, and the most accurate PTC tasting protocol was employed. Also, the percentage of such offspring is robust to choice of cutpoint and is not greatly reduced when the effects of age are accounted for or when young children are removed from the data set. Further, the proportions of nontaster offspring from the other mating types are also inconsistent with the predictions of the traditional one-locus recessive model.

Fifth, and most important for the model we are proposing, is the lack of information on other physiologic characteristics such as the types of salivary proteins present, the distribution of taste buds, and the tasting of other substances, particularly bitter substances such as quinine. It would be of interest to carry out a future study that would collect family data in which subjects are tested for ability to taste several substances in addition to PTC, along with other physiologic measures. This study should include repeat testing of taster offspring from NT $\times$ NT matings and more extensive determination of genetic marker phenotypes to test for nonpaternity, particularly for such taster offspring. Such a study would provide a more definite answer to the question of mode of inheritance for PTC taste deficiency as measured by the dilution method.

\section{ACKNOWLEDGMENTS}

We thank M. Anne Spence and D.C. Rao for helpful comments on previous versions of this manuscript. This research was supported in part by NIH grants R01 HD/AM-12252 and R29 GM-41440. Computing support for the project was provided by NIH Shared Instrumentation grant No. S10-RR-02882 and by the University of Michigan.

\section{REFERENCES}

Bartoshuk LM (1979): Bitter taste of saccharin related to the genetic ability to taste the bitter substance 6-n-propylthiouracil. Science 205:934-935.

Blakeslee AF (1932): Genetics of sensory thresholds: Taste for phenyl-thio-carbamide. Proc Nat Acad Sci USA $18: 120-130$.

Blakeslee AF, Salmon MR (1931): Odor and taste blindness. Eugen News 16:105-108.

Boyd WC, Boyd LG (1937): Sexual and racial variations in ability to taste phenyl-thio-carbamide with some data on the inheritance. Ann Eugen Lond 8:46.

Conneally PM, Dumont-Driscoll M, Huntzinger RS, Nance WE, Jackson CE (1976): Linkage relations of the loci for Kell and phenylthiocarbamide taste sensitivity. Hum Hered 26:267-271. 
Das SR (1956): A contribution to the heredity of the PTC taste character based on a study of 845 sib-pairs. Ann Hum Genet 20:334-343.

Das SR (1958): Inheritance of the PTC taste character in man: An analysis of 126 Rarhi Brahmin families of West Bengal. Ann Hum Genet 22:200-212.

Day NE (1969): Estimating the components of a mixture of normal distributions. Biometrika 56:463-474.

Essen-Moeller E (1938): Die Beweiskraft der Aehnlichkeit im Vaterschaftsnachweis. Theoretische Grundlagen. Mitteilungen d Anthropol Gesellsch Wein 68:9-53.

Fox AL (1931): Tasteblindness. Science 73:14.

Frank RA, Korchmar DL (1985): Gustatory processing differences in PTC tasters and non-tasters: A reaction time analysis. Physiol Behav 35:239-242.

Gent JF, Bartoshuk LM (1983): Sweetness of sucrose, neohesperidin dihydrochalcone, and saccharin is related to genetic ability to taste the bitter substance 6-n-propylthiouracil. Chem Senses 7:265-272.

Harris H, Kalmus H (1949): The measurement of taste sensitivity to phenylthiourea (PTC). Ann Eugen 15:24-32.

Harris H, Kalmus H (1951): The distribution of taste thresholds for phenylthiourea of 384 sib pairs. Ann Eugen 16:226--230.

Hartmann G (1939): Application of individual taste difference towards phenyl-thio-carbamide in genetic investigations, Ann Eugen 9:123-135.

Kalmus H (1958): Improvements in the classification of the taster genotype. Ann Hum Genet 22:222-230.

Kalmus H, Trotter WR (1962): Direct assessment of the effect of age on PTC sensitivity. Ann Hum Genet 26:145-149.

Lange K, Boehnke M (1983): Extensions to pedigree analysis. V. Optimal calculation of Mendelian likelihoods. Hum Hered 33:291-301.

Lange K, Boehnke M, Weeks D (1986): Programs for pedigree analysis. Unpublished computer program documentation.

Lange K, Weeks D, Boehnke M (1988): Programs for pedigree analysis: MENDEL, FISHER and dGENE. Genet Epidemiol 5:471-472.

Lee BF (1934): A genetic analysis of taste deficiency in the American Negro. Onio J Sci 34:337-342.

Lugg JWH, Whyte JM (1955): Taste thresholds for phenylthiocarbamide of some population groups. I. The thresholds of some civilized groups living in Malaya. Ann Hum Genet 19:290--311.

Merton BB (1958): Taste sensitivity to PTC in 60 Norwegian families with 176 children. Confirmation of the hypothesis of single gene inheritance. Acta Genet Basel 8:114-128.

Rao DC, Morton NE (1977): Residual family resemblance for PTC taste sensitivity. Hum Genet 36:317-320.

Reddy BM, Rao DC (1989): PTC taste sensitivity revisited: Complete sorting test supports residual family resemblance. Genet Epidemiol, 6:413-421.

Roche AF, Chumlea WC, Thissen D (1988): Assessing the skeletal maturity of the hand-wrist: FELS method. Springfield, IL: Charles C. Thomas.

Self SG, Liang KY (1987): Asymptotic properties of maximum likelihood ratio tests under nonstandard conditions. J Am Stat Assoc 82:605-610.

Snyder LH (1931): Inherited taste deficiency. Science 74:151-152.

Snyder LH (1932): The inheritance of taste deficiency in man. Ohio J Sci 32:436-440.

Spence MA, Falk CT, Neiswanger K, Field LL, Marazita ML, Allen FH, Siervogel RM, Roche AF, Crandall BF, Sparkes RS (1984): Estimating the recombination frequency for the PTC-Kell linkage. Hum Genet 67:183--186.

Edited by Jean W. MacCluer 